\title{
Object Segmentation based on Shape Feature using Active Contour Model
}

\author{
Snehal V. Talikoti \\ P.G Student \\ Department of Computer Engg, \\ Late G.N. Sapkal College of Engineering, \\ Anjaneri, Nashik
}

\author{
J.V. Shinde \\ Assistant Professor \\ Department of Computer Engg, \\ Late G.N. Sapkal College of Engineering, \\ Anjaneri, Nashik
}

\begin{abstract}
Active contour models has already under study from the past many years, several methods have been proposed for forming contours. Active contour are the curves that are generated by the computer that move within an images to find boundaries of the object in an image. They are also used in image analysis and computer vision to recognize and find objects, and to describe their shape. In the proposed system Active Contour Model that segments one or many regions of the image that are visually alike to an object of interest said as prior. The probability density function is used for extracting the color feature by applying heuristic rule, and then the new proposed shape detection algorithm is used to detect the photometric feature shape of the object in an image. For accurately segmenting object in an image. The proposed system provides the accurate results on real world and synthetic datasets.
\end{abstract}

\section{General Terms}

Algorithms, Experiments, Results.

\section{Keywords}

Active contour model, Probability density function and heuristic rules.

\section{INTRODUCTION}

Segmentation is the procedure of separating a computerized picture into various portions (sets of pixels or ples, are also called as super pixels). The point of segmentation is to clear up and/or change the description of a picture into something which is more significant and easy to review. Each of the pixels in a locale are same as for certain characteristics or set of image attribute called contour, for example, shape, intensity, color. The curve called an active contour that dynamically grows in the image domain toward the object boundary, there are two types of forces that are internal and external forces.

- Internal forces are used to find the geometric properties of the curve, such as smoothness or stiffness.

- External forces based on the image features used for the segmentation, such as color, shape intensity or texture.

An Active contour model that searches for an input image is a view of known object, called prior. Prior is a part of the image to be detected from an initial image. Prior images are also said as logo, a face, or a home object. Initial image contain real world or synthetic images. It has many objects to be matched with the prior image. Use PDF photometric feature as color and shape. Compute the accuracy of the initial image to the prior by coinciding between their probability density function. The heuristic law converge or diverge and it will decide either to include or exclude the pixels present in an image. Heuristic law are generally created by user to get faster results. Sometimes overlapping is not possible, because the prior may not be copy of initial image, or depends on camera, small difference in intensity or it may contain noise.

Color is widely used to detect image because color is invariant among the other features. But if the objects and its background are of same color then it will extract the contours with similar color. [1]

To overcome this problem we are using the both the features color as well shape.

The main aim is to extract the object from initial image by using the prior image, to extract the color and shape features of the object accurately.

\section{LITERATURE SURVEY}

The concept of active contours was first introduced by Kass were originally boundary methods, Snakes: Active Contour Models. A snake is a parametric curve which tries to move into a position where its energy minimization is performed. [2]

$$
\mathrm{Es}_{\text {nake }}=\mathrm{E}_{\text {int }}+\mathrm{E}_{\text {ext }}+\mathrm{E}_{\text {con }}
$$

Internal Energy (Eint) relies on upon the inner properties of the curve and is the sum of elastic energy and bending energy. External energy (Eext) of the contour is derived from the image itself, so that it takes on its smaller values at the function of interest such as boundaries. Curve should be placed manually near the object.

In the classical active contour models due to Kass et al, discovered some drawbacks.

There are two drawbacks:

1. Its failure to identify images with nonconvex objects and

2. Its sensitivity to initialization.

Chan and Vese et al, proposed new model for active contour to find the objects in the given image [3], it uses some techniques of curve evolution, level set function, and Mumford shah function for image segmentation. These function are used to minimize the energy functional. The minimization of the energy functional in active contour models was computationally tough

This paper presents the relationships between the boundary and region functional. [8] It uses Green Riemann theorem, corresponding Euler Lagrange equations are derived and used to define scheme to evolve the initial region. The problem of the existence and uniqueness of a solution to Probability distribution equation has not been addressed. Rederive 
equation to solve to problems in simple and easy way. Here they addressed only on the boundary and the region functions.

All the above methods are developed energy minimization scheme for generating the contours.

Many active contour methods work on the gray scale images,

To extract the contours in an image, mainly used in medical science, examples MRI, CT images.[4]

In this method [1] the color images are used to find the object and segment that object using ACM

\section{SYSTEM ARCHITECTURE}

The proposed system comprises preprocessing, contour extraction, PDF extraction, shape detection. Fig. 2 shows the system architecture of the proposed system.

Firstly it takes two images as input from the image dataset, First image is said as initial image, and the second image is the part of the initial image said as prior, then image is preprocessed using gradient vector and Gaussian filter. Contours are extracted from the image accurately. For contour extraction active contour method is used. After extraction of contours, by the original images RGB values are calculated by using probability density function for both the images. Apply the shape extraction algorithm to detect accurate object present in an image, using both the features colour and shape. Accurate object is segmented by varying the threshold value because every image differs from one another, its intensity values are different

The proposed methodology has been divided into the under explained following stages:-

\subsection{Contour model}

In this module the preprocessing of image is done by using gradient vector and Gaussian filter. Gaussian filter is used to remove noise and smoothen the image, by doing this will extract the sharp edges in an image. Apply the contour algorithm on both the prior and initial image, it starts extracting the contour boundaries, if they nearest and of the given object, it will form contour, otherwise it discards those points which are furthest. Likewise the contours are formed for both the prior and initial images.

The pseudocode for algorithm1 is as follows:-

Input: Initial image, prior image.

\section{Output: Extract Contour}

Parameter initialization: points in matrix $\left\{\mathrm{x} \ldots \mathrm{x}_{\mathrm{n}}\right\}$; Function of points $\{f(x), f(x-h), f(x+h)\}$; step size $h$; maximum step sixe $\mathrm{H}$; point exist on map A; Threshold $=60$.

1. Compute gradient vector $\nabla \mathrm{F}(\mathrm{x})$ using

$$
\nabla \mathrm{F}(\mathrm{x})=\left[\begin{array}{c}
\frac{\delta f(x)}{\delta x_{1}} \\
\frac{\delta f(x)}{\delta x_{2}} \\
\vdots \\
\frac{\delta f(x)}{\delta x_{n}}
\end{array}\right]=\mathrm{g}(\mathrm{x})
$$

2. Apply Gaussian filter, Calculate $\mathrm{F}(\mathrm{g}(\mathrm{x}))$

3. Compute $F(x), F(x+h)$ and $F(x-h)$

4. If $\mathrm{F}(\mathrm{x})<\mathrm{F}(\mathrm{x}+\mathrm{h})$, Evaluate $\mathrm{F}(\mathrm{x}-\mathrm{h})$

5. If $F(x-h)=F(x)>$ threshold, Select point

6. To validate the curve, Compute turning point $x$ by using, $\mathrm{F}(\mathrm{x})=\mathrm{F}(\mathrm{x}-\mathrm{h})=\mathrm{F}(\mathrm{x}+\mathrm{h})>$ threshold, Select point

For selection of contour, If $\mathrm{x}$ a minimum point and

$$
\left|x-X_{n}\right|>\operatorname{minSize} \text {, again go to step 5, else continue. }
$$

7. If $\left|x-X_{n}\right|<e$ then

8. $\mathrm{F}\left(\mathrm{A}^{*}\right)=\min \left[\mathrm{F}(\mathrm{A}), \mathrm{F}\left(\mathrm{A}_{\mathrm{n}}\right)\right]$ and $\mathrm{STOP}$, else continue.

9. Discard point with highest $F$ value and replace it by $A$; go to Step 5

\subsection{Probability Density Function}

The PDF of prior and initial region must be calculated based upon color such as red, blue and green. The pdfs of initial region evolves around the region by heuristic rules. For Calculation of Probability density function this equation is used

$$
P d f\left(\Omega_{p, q}\right)=\frac{1}{\sigma \sqrt{2 \pi}} e^{\frac{-(x-m)^{2}}{2 \sigma^{2}}}
$$

Here $\Omega$ is the input region, $\mathrm{p}$ is prior, $\mathrm{q}$ is evolving region, $\mathrm{x}$ be the current value of pixel, and $m$ be the mean of RGB colors.

Heuristic rule: Calculate PDF of initial image and PDF of prior image overlap them, if the value is 1 , then PDF of both should be equal to zero,

There exist 3 conditions

1. If PDF of initial image $>1$ then PDF of initial image is greater than that of PDF of prior image, then converge to exclude those points.

2. If PDF of initial image $<1$ then PDF of initial image is smaller than that of PDF of prior image then diverge to include those points.

3. If $\mathrm{PDF}$ of initial image $=1$ then $\mathrm{PDF}$ of initial image and PDF of prior image are equal.

\subsection{Shape Extraction}

By using all the above modules we get contours and PDF of color, by using them calculate the accurate shape of the object based color and shape.

After getting contours of prior and evolving region compare them, assign pixels as 1 if that contain points of object. Compare the values of color PDFs, if that are within the threshold the accurate object is retrieved.

The pseudocode for algorithm 2 is as follows:-

Input: Contours of prior and initial image.

Output: Displaying the segmented object (O)

Parameters: Prior p; initial image q; Contour $c_{p}$ in $\mathrm{p}$; Contour $c_{q}$ in q; counter; variable val; $\mathrm{p} 1, \mathrm{q} 1$ as rectangle; threshold $=$ $60 ; \mathrm{x}$ and $\mathrm{y}$ are pixel coordinates.

1. Initialize $\left(c_{p}, c_{q}\right)$

2. Compute val=compare $\operatorname{shape}\left(c_{p}, c_{q}\right)$

3. Assign each point in $c_{p},\left(c_{p} \mathrm{x}, c_{q} \mathrm{y}\right)=1$, each point in $c_{q},\left(c_{p} \mathrm{x}, c_{q} \mathrm{y}\right)=1$ 
4. Compute $\mathrm{x}$ and $\mathrm{y}$ as

For each $(\mathrm{x}=0 ; \mathrm{x}<$ width; $\mathrm{x}++)$ and $(\mathrm{y}=0 ; \mathrm{y}<$ height; $\mathrm{y}++)$

5. $\operatorname{If}(\mathrm{p} 1(\mathrm{x}, \mathrm{y})==1 \& \& \mathrm{q} 1(\mathrm{x}, \mathrm{y})==1)$ then

Counter $=$ Counter +1 ;
6. Compute Total =cp.counter; val=total/counter;

7. Check (val $>$ threshold)

8. Compute val as val=compare $\left(\operatorname{pdf}\left(c_{p}\right), p d f\left(c_{q}\right)\right)$;

9. Check (val $>$ threshold) //Add to match points

10. Display O

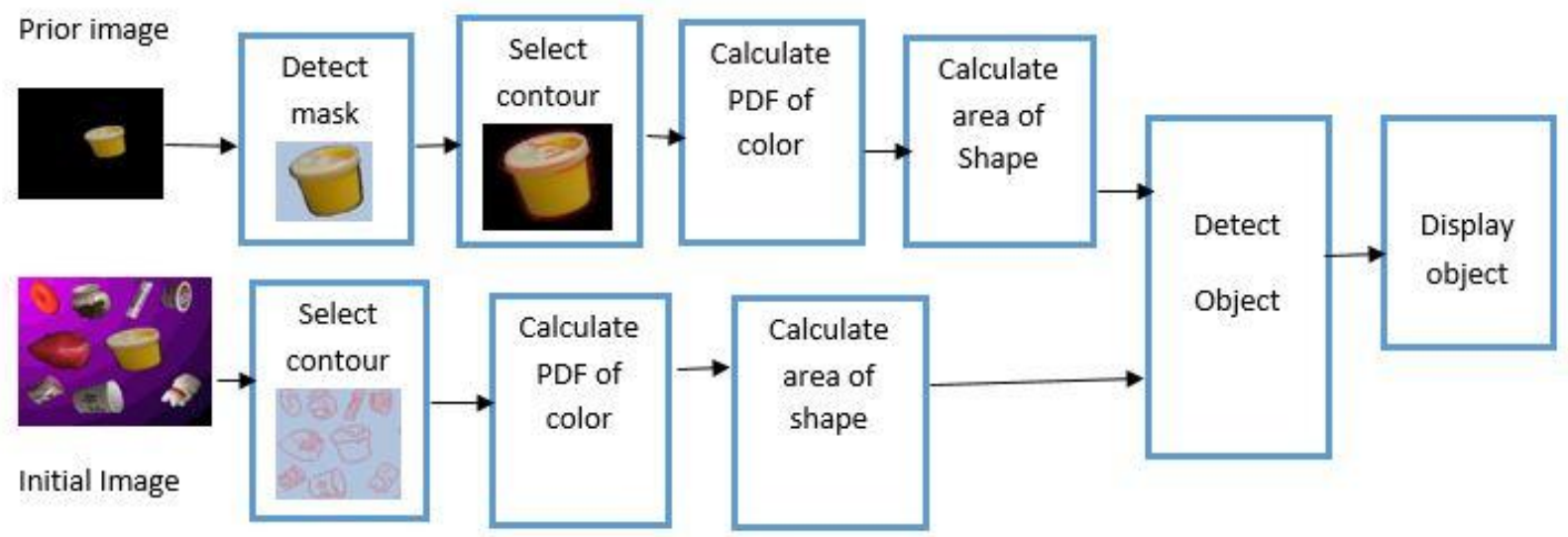

Fig 1: Proposed system Architecture

\section{EXPERIMENTAL RESULTS}

\subsection{Experimental setup}

The algorithm is implemented in .Net Framework 3.5 using c\#. To verify the result of the proposed system it uses overlap index. Here ground truth 100-for COIL [13] is used to show the performance of system. Fig 2. Shows results of proposed system with different images.

\subsection{Dataset}

\subsubsection{Ground Truth 100-forCOIL}

It is a synthetic database, the database has 100 images displaying some objects of COIL-100 database. It displays Background against non-uniform form and it contains many objects in a single image of size $484 \times 484$. [13]

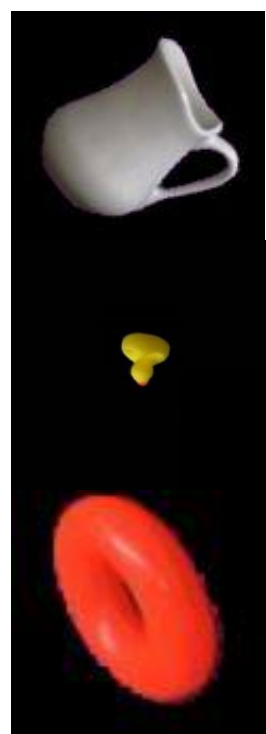

(a)

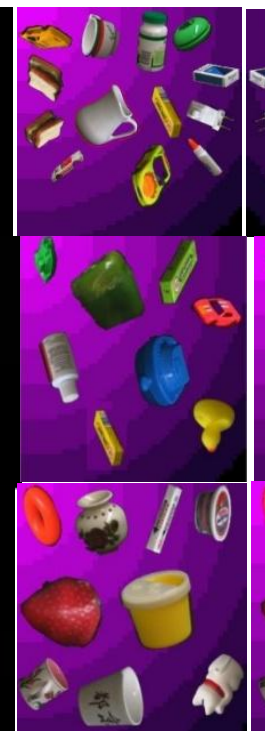

(b)

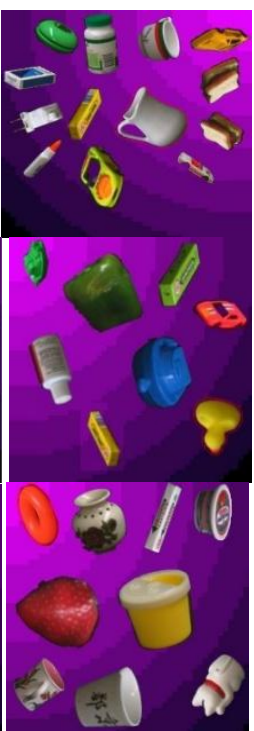

(c)
Fig 2: (a) Prior image; (b) Initial image; (c) Result of proposed system(color and shape feature)

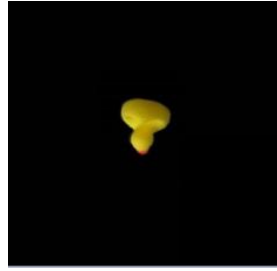

(a)

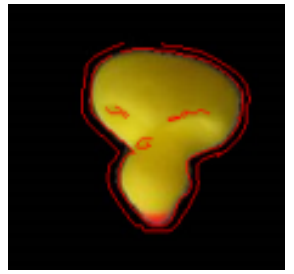

(b)
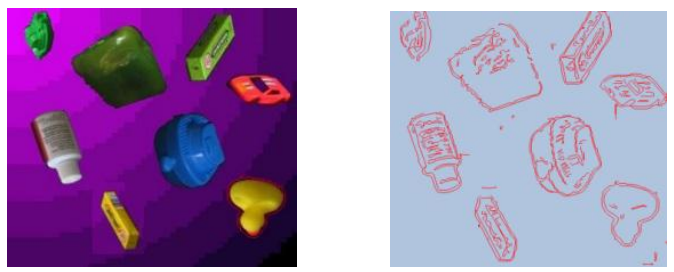

(c)

(d)

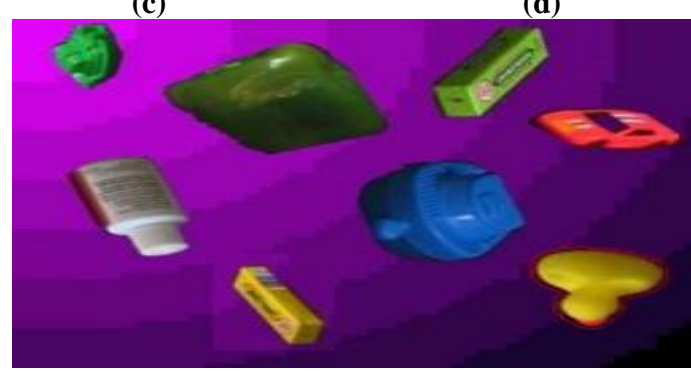

(e)

Fig 3: Screenshot of proposed system Implementation

(a) Prior image; (b) Contour of prior image; (c) Initial image; (d) Contour of Initial image; (e) Result of proposed system(color and shape feature)

Figure 3 shows the result of the proposed system (e) depicts the segmented object in red boundary.

\subsection{Performance Evaluation}

Accuracy of the system is measured by overlap index as follows: 


$$
X\left(G, \Omega^{*}\right)=\frac{\operatorname{Area}\left(G \cap \Omega^{*}\right)}{\operatorname{Area} G \cup \Omega^{*}}
$$

Let $\mathrm{O}$ be the object view to be detected in an input image I,

Let $\mathrm{G}$ be the prior image (part of actual image) depicting the object $\mathrm{O}$

Where $\boldsymbol{\Omega}^{*}$ is the segmented region by our algorithm based on shape and color.

The overlap index $\mathrm{X}$ should be between [0.1], and closer the $\mathrm{X}$ to 1 , the better the accuracy in detection is.

Also compute the percentage I of pixels of G belonging to $\boldsymbol{\Omega}^{*}$ :

$$
I\left(G, \Omega^{*}\right)=\frac{\operatorname{Area}\left(G \cap \Omega^{*}\right)}{\operatorname{Area}(G)}
$$

An object view $\mathrm{O}$ is missed if it is depicted in the input image I but it has not been detected i.e. $X\left(\boldsymbol{G}, \boldsymbol{\Omega}^{*}\right)=0$. And $\mathrm{M}$ denote the percentage of missed object view.

\subsection{Results}

\subsubsection{Result table without pose change}

The results shows the value of images from the ground-truthfor-coil-100 database [13]. The first column shows the image numbers from the database. Second column shows the results of existing system based on object segmentation based on color, the object segmentation more difficult, because often the background includes parts visually similar to the object to be detected and also displays the value of $\mathrm{X}$ and I. To overcome this problem the third column shows the object segmentation based on shape and color feature and the value increases compared to the existing system.

Table 1. Result of earlier and proposed system

\begin{tabular}{|c|c|c|c|c|}
\hline \multirow{2}{*}{ Image no. } & \multicolumn{2}{|c|}{ Existing } & \multicolumn{2}{c|}{ Proposed } \\
\cline { 2 - 5 } & $\mathrm{X}$ & $\mathrm{I}$ & $\mathrm{X}$ & $\mathrm{I}$ \\
\hline 851 & 0.3882 & 0.4657 & 0.60533 & 0.8138 \\
\hline 56 & 0.3391 & 0.4245 & 0.7688 & 0.9629 \\
\hline 85 & 0.4072 & 0.4763 & 0.7092 & 0.8664 \\
\hline 92 & 0.5303 & 0.5619 & 0.8180 & 0.8734 \\
\hline 94 & 0.3934 & 0.4288 & 0.6753 & 0.9260 \\
\hline
\end{tabular}

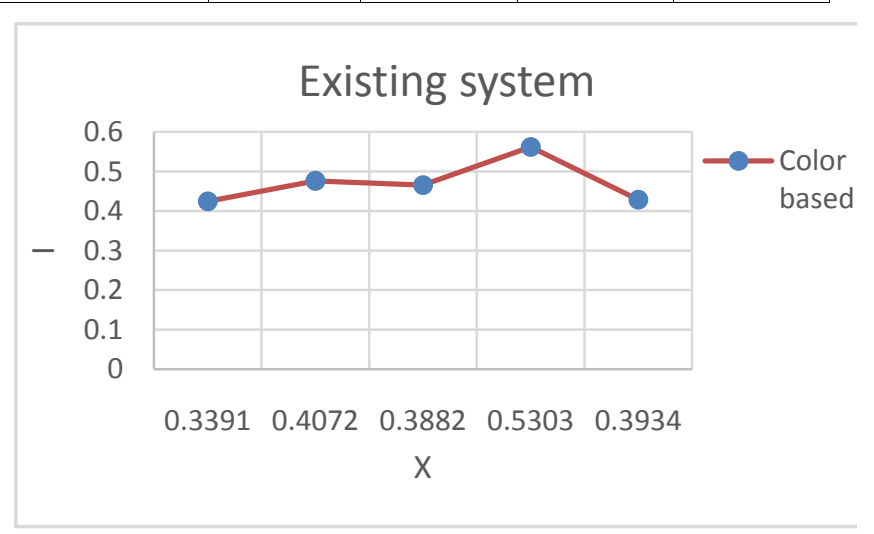



Fig. 4: Graph (a) earlier system based on color; (b) proposed system based on color and shape feature

Figure 4 shows the graph of existing system used with color feature and proposed system used with the shape and color as a feature.

\subsubsection{Robustness against Pose change}

Table 2 shows the overlap index obtained. This table also reports the accuracy output from the segmentation of the image. The prior image is given for the poses rotated of 5 degrees. $\mathrm{M}$ be the missed part which is 0 . The value of $\mathrm{X}$ and I decreases with respect to change in pose.

Table 2. Result of earlier and proposed system

\begin{tabular}{|c|c|c|c|}
\hline \multirow{2}{*}{ View[degree] } & \multicolumn{3}{|c|}{ Proposed } \\
\cline { 2 - 4 } & $\mathrm{X}$ & $\mathrm{I}$ & $\mathrm{M}$ \\
\hline 5 & 0.7677 & 0.9588 & 0 \\
\hline 10 & 0.7318 & 0.9361 & 0 \\
\hline 15 & 0.7538 & 0.9185 & 0 \\
\hline 20 & 0.427 & 0.541 & 0 \\
\hline
\end{tabular}

\section{CONCLUSION AND FUTURE SCOPE}

This paper describes active contour technique for generating contours. Region based ACM that segments image regions that are visually alike to an object of interest called prior or trained dataset. It contains the object to be segmented from an image. That is the prior and evolving region are described by probability density function (PDF) of a photometric feature as shape. PDF function is used to generate the RGB values of the contours, Heuristic rules are applied to find the contour regions which are alike to the object. The shape extraction algorithm is used to detect accurate shape of the object based on color and shape feature. This research lead us towards improving the accuracy of the object segmentation based up on shape. Future work will include the development of object segmentation with short period of time with the rotation.

\section{ACKNOWLEDGMENTS}

My deepest gratitude goes to my guide and PG coordinator Prof J .V. Shinde your help has been invaluable, as a Professor. Thank you for encouragement, and directing them my way. Thanks to all the staff members of the college. 


\section{REFERENCES}

[1] Michela Lecca, Stefano Messelodi, Raul Paolo serapioni. "A New Region Based Active Contour Model for Object Segmentation" Springer, J Math Imaging Vis 2015

[2] Kass, M.,Witkin, A.,Terzopoulos,D.: Snakes: active contour models. Int. J. Comput. Vis. 1(4), 321-331 (1988)

[3] Chan, T., Vese, L.: Active contours without edges. IEEE Trans. Image Process. 10(2), 266-277 (2001)

[4] Chakraborty, A., Staib, L., Duncan, J.: Deformable boundary finding in medical images by integrating gradient and region information. IEEE Trans. Med. Image 15(6), 859-870 (1996)

[5] Darolti, C., Mertins, A., Bodensteiner, C., Hofmann, U.G.: Local region descriptors for active contours evolution. Trans. Image Proc. 17(12), 2275-2288 (2008)

[6] Paragios, N., Deriche, R.: Geodesic active regions and level set methods for supervised texture segmentation. Int. J. Comput. Vis. 46(3),223247(2002)

[7] Ronfard, R.: Region-based strategies for active contour models. Int. J. Comput. Vis. 13, 229251 (1994)

[8] Jehan-Besson, S., Barlaud, M., Aubert, G., Faugeras, O.: Shape gradients for histogram segmentation using active contours. In: Proceedings of International Conference on Computer Vision, pp.

[9] Chan, T., Vese, L.: Active contours without edges. IEEE Trans. Image Process. 10(2), 266277 (2001)

[10] Darolti, C., Mertins, A., Bodensteiner, C., Hofmann, U.G.:Local region descriptors for active contours evolution. Trans. Image Proc. 17(12), 22752288 (2008)

[11] Atkinson, K., Han, W.: Theoretical Numerical Analysis. A Functional Analysis Framework, Texts in Applied Mathematics, vol. 39. Springer, New York (2009)

[12] Lecca, M.,Messelodi, S.: Rotation, rescaling and occlusion invariant object retrieval. In: Rajpoot, N., Bhalerao, A. (eds.) British machine vision conferenceBMVC, BMVAPress, pp. 14.1-14.10 (2007)

[13] FBK-TeV: Technologies of vision-Fondazione Bruno Kessler, masks of coil-100 dabatabase. https://tevstatic.fbk.eu/DATA BASES/coil-100-masks.tgz (2006)

[14] He, L., Peng, Z., Everding, B., Wang, X., Han, C.Y., Weiss, K.L., Wee, W.G.: A comparative study of deformable contour methods on medical image segmentation. Image Vis. Comput. 26(2), 141-163 (2008) 